\title{
Buchbesprechungen - Book Reviews - Llvres Nouveaux
}

Deucher I Miller: Der operierte Magen. Bibliotheca Gastroenterologica, Fasc. 6. Kar-ger, Basel/New York 1964. XX + 276 S. 92 Abb. 65 Tab. Preis: sFr./DM 65.-.

Die hier zusammengefaßten Vorträge wurden an der Jahresversammlung der Schweizerischen Gesellschaft für Gastroenterologie in Solothurn vom 24.-26. Okto-ber 1963 gehalten.

Hauptthemen waren: Operationsmethoden, die Spätresultate, Untersuchung des operierten Magens, die Syndrome nach Magenresektion und die Therapie des operierten Magens. Die Thematik geht aus von den durch Haemmerlí dargelegten physiologischen Grundlagen der Resektionstherapie bei gastroduodena-lem Ulkus. Hiernach ist die logische Operationsmethode die vollständige Vagotomie mit Antrumresektion. Vagale und antrale Phasen der das peptische Geschwür ver-ursachenden Sekretion werden hierdurch beeinträchtigt. Die Antrumresektion plus Vagotomie (A V-Resektion) haben Nietlíspach u. Mitarb. mit gutem Erfolg praktiBuchbesprechungen - Book Reviews - Livres Nouveaux 313 ziert. Möglicherweise ist dieses Verfahren den alten Methoden Billroth I und Billroth II überlegen. Níssen sowie Berchtold berichteten über ante- und retrokolische GE bei Billroth II. Die Spätresultate waren insgesamt günstig, wenngleich nicht verkannt wurde, daß der Chirurg weniger Mißerfolge als der Internist zu sehen bekommt. Salzer hob hervor, daß die Karzinomgefährdung des Magenresezierten - was infolge der chronischen Gastritis denkbar ware - nicht höher ist als die des nicht Operierten. Miller u. Mitarb. stellten in einer Gemeinschaftsuntersuchung fest, daß das befriedi-gende oder unbefriedigende Endresultat einer Magenresektion nicht nur von einer korrekten Indikationsstellung sondern auch vom Können des Operateurs abhängt. Wesentlich für den Erfolg des Eingriffes ist aber auch die Persönlichkeit des Operierten selbst, der nicht Heilung ohne sein Zutun fordern darf (Mangold). Oft lassen sich Abweichungen von der Norm objektivieren, obwohl der Patient beschwerdefrei ist (Scholer u. Mitarb.). Zu den wichtigsten postoperativen Komplikationen gehören das Ulkusrezidiv (Rudler), das Dumping-Syndrom (Kellner), für dessen Entstehen oder Ausbleiben ebenfalls die Struktur des Kranken von Bedeutung ist, und das blind-loop-Syndrom, welches zusammen mit dem Syndrom der zuführenden Schlin-ge Mangelzustände (Maldigestion und Malabsorption) erzeugen kann (Hotz). Man-gelanämien nach Magenresektion beruhen vorwiegend auf einem Defizit an Eisen (Moeschlin u. Mitarb.). HC1- und Vitamin C-Gabe können diesen Zustand bessern, wobei die Salzsäure ein Ulkusrezidiv nicht fördern soil. Leuthold u. Mitarb. berichteten über die Dünndarmbiopsie nach Magenresektion, wobei sie zu der interessanten Feststellung kamen, daß sich im magennahen Jejunum zwar Zottenverplumpungen finden, diese aber etwa 30 $\mathrm{cm}$ aboral der Anastomose nicht mehr nachweisbar seien. Auch Heínkel berichtete, daß die Jejunitis nach Magenresektion wohl sehr viel sel·te-ner sei, als angenommen würde. Leuthold schließt aus seinen Befunden, daß die Malabsorption bei der Genese des PostgastrektomieSprue-Syndroms keine Rolle spielen dürfte. Über die noch immer nicht endgültig geklärte Frage, ob die Magenresektion Leberschäden verursache, berichteten Kalk im bejahenden, Berndt $u$. Mitarb. im verneinenden Sinne. Über Realimentation und Diätüberwachung sprach Demole, zum 
Ende der Chirurg Deucher über die Möglichkeiten von Korrekturen, nämlich über Operationen am operierten Magen.

Die Schlußfolgerung des Kongresses lautete, daß der operative Eingriff die derzeit immer noch beste Methode sei, um das resistente peptische Geschwür zu behandeln. Diese Feststellung schließt nicht aus, daß die Operationsmethoden weiterhin verbessert werden sollten, mit dem Ziel, die Rezidivquote und die postoperativen Störungen zu verringern. - Zusammenstellung und Qualität der Vorträge bestätigen das hohe Niveau der Schweizerischen Gesellschaft für Gastroenterologie und ihrer Gäste. Der Karger-Verlag legt in bewährter Weise ein drucktechnisch ausgezeichnetes Buch in die Hände der gastroenterologisch interessierten Leserschaft, das den Bogen zwischen moderner Physiologie und Praxis spannt. L. Demlíng, Stuttgart Caroli, J. et Corcos, V.: Maladies des voies biliaires intrahépati«jues segmentaires. Masson, Paris 1964. 352 p. 93 fig. Prix: F 76.-.

Les voies biliaires «intrahépatiques» étudiées ici sont uniquement les canaux segmentaires, à $\Gamma$ exclusion des canalicules intra-, péri- et. interlobulaires d'une part et des canaux hépatiques gauche et droit dans la hile. Les premiers font partie de la pathologie interne, les seconds regardent les chirurgiens, et entre deux s'étendait jusqu'ici un no-man's land qui fait $\Gamma$ objet de ce volume.

314 Buchbesprechungen - Book Reviews - Livres Nouveaux Après des chapitres d'embryologie, d'histologie, de physiologie et d'anatomie, les A. décrivent la radíologíe des voies biliaires intrahépatiques par cholangiographie veineuse à la morphine (pour bloquer le sphincter d'Oddi) ou par injection directe du produit de contraste dans les canaux dilates (quand la clearance hépatique est mauvaise), examen utilement complete par tomographie; en accentuant le contraste par le logetron, on obtient d'excellentes images des voies biliaires intrahépatiques. En principe, un dessin de ces canaux trop précoce et trop bien opacifié par rapport à celui de la voie biliaire principale est un signe de sténose organique incomplete dans le bile.

Les chapitres suivants commencent par les observations personnelles, puis celles de la littérature, avant de brosser le tableau clinique des différentes maladies des voies biliaires intrahépatiques et d'en discuter le diagnostic différentiel et la théra-peutique. Les affections congénitales (dilatations ou agénésies), tumorales (papil-lomes, fibromes, cancers) et les différents types de lithiase sont passes en revue avant une etude du retentissement sur les voies biliaires intrahépatiques des kystes, abcès ou tumeurs du foie.

II n'est guère possible d'analyser chaque paragraphe, car on ne resume pas des observations aussi fouillées au texte dense et encore moins la centaine de cliches, minutieusement interprétés, qui illustrent les documents cliniques. Présenté avec le soin et la clarté typographique habituels de Гéditeur Masson, ce volume fera date dans $\Gamma$ étude de syndromes encore trop méconnus. II fallait Гexpérience exceptionnelle et la richesse de documentation de Carolí et de $\Gamma$ Ecole de Saint-Antoine pour une telle monographie, à laquelle se rapporteront désormais médecins, chirurgiens et spécialistes en face de ces cas limites. M. Demole, Geneve Bourne, G. H. and Wilson, E. M. H.: World Review of Nutrition and Dietetics. Vol. 5. S. Karger, Basel/New York 1965, 381 p. 24 Abb. 23 Tab. Price: sFr./DM 69.-

Ce volume est un de ces «mélanges» qui paraissent curieusement appréciés de nos jours - ne sommes-nous vraiment plus capables de lire un livre consacré à un seul sujet ? - compose de 2 parties bien distinctes. 
Les 4 premiers exposes, rédigés par des diététiciennes éminentes et anglo-saxonnes, sont consacrés à $\Gamma$ histoire de la nutrition et de la diététique, ou à $\Gamma$ application pratique de celle-ci. Deux travaux concernent la vitamine-A et son métabolisme, particulièrement Гinfluence des protéines sur son absorption (S. Mahadevan et coll., Bangalore). Un autre relate les elements sur la base desquels on a établi les dernières « Recommended protein allowances» (E. G. Holmes, Adelaide). De son côté, R. J. C. Steward (Londres) présenté une revue très complete de la «pathologie osseuse dans la malnutri tion expérimentale». Le volume se termine par une etude fort originale de H. E. Ganther (Madison) sur le métabolisme du Selenium chez Гanimal: ce mineral connu pour sa toxicité a été explore maintenant grace aux isotopes, et peut avoir un effet utile dans certaines rations, à la dose de 0,04 p. p. m. M. Demole Aguercíf, Méziane: Contribution à I'étude de la forme anémique de la hernie de l'estomac par l'hiatus æsophagien du diaphragme chez Tadulte. These de Geneve No. 2905,106 p. (1963). Le contenu de cet important travail dépasse de beaucoup son titre, car c'est une Buchbesprechungen - Book Reviews - Livres Nouveaux 315 revue générale de la hernie hiatale et de son traitement, avec la statistique de la Clinique Chirurgicale de Geneve et des résultats obtenus par sa correction opératoire.

L'anémie (hémoglobine au-dessous de 75\%) affecte la moitié des hernies hiatales, mais elle est rarement (4 fois sur 58 cas) inférieure à $50 \%$ Hgb. Dans la présente série, les symptômes révélateurs de la hernie étaient plus souvent d'ordre digestif que des manifestations de l'anémie. L'anémie est de type légèrement hypochrome et la recherche du sang occulte dans les selles toujours positive une fois ou $\Gamma$ autre, si on la recherche avec perseverance. II s'agit en effet toujours d'une soustraction chronique de sang, soit par cesophagite peptique (due au reflux), soit par gastrite erosive (par gene mécanique de la circulation de retour), soit encore par ectasies veineuses de la paroi gastrique. Si un trouble de Гabsorption du fer semble improbable, on peut en revanche faire jouer un role à $\Gamma$ insuffisance d'apport alimentaire du fer ou une diminution des reserves de fer (la hernie hiatale survenant 3 fois sur 4 chez des femmes).

L'anémie de la hernie hiatale répond bien - mais temporairement - à la thérapeu-tique martiale, et ne se reproduit plus après cure chirurgicale de la lesion.

M. Demole

VARIA

Heinz Karger Memorial Prize

The Dr. Heinz Karger Memorial Foundation in Basel announced last year an international competition for outstanding medical-scientific papers, to be written on the subject "Enzymology of Leukemic Cells". The Council of the Foundation -which comprises Professor H. Lüdín, Basle, Professor G. Mayor, Zurich, Dr. R. Ríchterích, Berne, Professor J. R. Rüttner, Zurich, and the publisher Mr. T. Karger -has now awarded the sFr. 3000.- prize to the two American research workers Dr. J. B. Block and Dr. S. L. Bonting of the National Institutes of Health, Bethesda, Md., for their paper about "ATPase Transport in Normal and Leukemic Leukocytes". This paper will be published in the international Journal Enzymologia bíologica et clinica. Highly recommended were the papers submitted by Dr. W. Wílmanns of Tubingen (awarded the second prize) and Prof. D. Dioguardi of Cagliari and Prof. A. Agostini of Milano (awarded the third prize).

The Council of the Foundation has also announced that the subject of the second competition, to be held next year, will be "Microangiological Problems in Arteriosclerosis". 